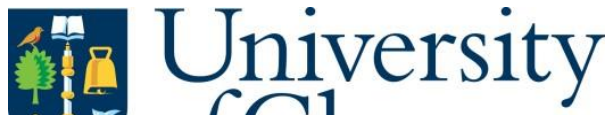 of Glasgow
}

Fan, H., Zhang, J., Zuo, S., Hu, Q., Feng, Q. and Heidari, H. (2021) A CMOS hall sensor modelling with readout circuitry and microcontroller processing for magnetic detection. Review of Scientific Instruments, 92(3), 034707. (doi: $\underline{10.1063 / 5.0038295}$ )

The material cannot be used for any other purpose without further permission of the publisher and is for private use only.

There may be differences between this version and the published version. You are advised to consult the publisher's version if you wish to cite from it.

http://eprints.gla.ac.uk/234272/

Deposited on 15 February 2021

Enlighten - Research publications by members of the University of Glasgow http://eprints.gla.ac.uk 


\title{
A CMOS Hall Sensor Modelling with Readout Circuitry and Microcontroller Processing for Magnetic Detection
}

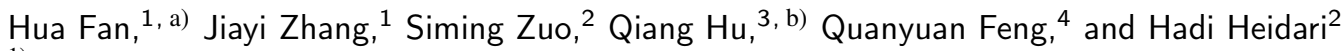 \\ ${ }^{1)}$ School of Electronic Science and Engineering, University of Electronic Science and Technology of China, Chengdu 610054, \\ China. \\ 2) James Watt School of Engineering, University of Glasgow, G12 8QQ, Glasgow, United Kingdom. \\ 3) Jihua Laboratory, Foshan 528200, China. \\ ${ }^{4)}$ School of Information Science and Technology, Southwest Jiaotong University, Chengdu 611756, \\ China.
}

(Dated: 15 February 2021)

\begin{abstract}
A Hall sensor array system for magnetic field detection and analysis is realized in X-FAB $0.18 \mu \mathrm{m}$ CMOS technology. Magnetic field detection is attributed to the magnetization of metal coils to metal particles and the sensing characteristics of Hall sensor array. The system put forward a complete solution from Hall sensors, analog front-end circuit (AFE), analog-to-digital converter (ADC) to Microcontroller Unit (MCU). Using Ansoft Maxwell and COMSOL Multiphysics software for simulation verification, the minimum diameter of magnetic particles that can be detected in the system is $2 \mu \mathrm{m}$. The measured SNDR, SFDR and ENOB of the proposed ADC are $70.61 \mathrm{~dB}, 90.08 \mathrm{~dB}$ and 11.44-bit. The microsystem based on STM32 combines hardware and software design, which can effectively adjust the motion parameters and realize the real-time display in the LCD screen of the magnetic field and voltage information. Compared to the prior system, the portability, cost and efficiency have been considerably improved, which is aimed at the rapid measurement of heavy metal particles such as $\mathrm{Fe}, \mathrm{Co}, \mathrm{Ni}$, etc. in ambient air and blood.
\end{abstract}

\section{INTRODUCTION}

In recent years, with the acceleration of the construction of industrialized cities and the rapid economic development, people's quality of life is facing challenges at the same time ${ }^{1}$. Due to the influence of large-scale industrial production and unreasonable exhaust emissions, the types and concentrations of particulate matter in ambient air are increasing year by year. In addition to the common atmospheric particles, PM 2.5 , the pollutants in the air also contain heavy metals. Research shows that if human beings are in the environment of air pollution for a long time, the accumulation of heavy metals in the respiratory system will affect the lung and other organ$\mathrm{s}$, resulting in serious systemic diseases such as pulmonary failure $^{2}$. If magnetic metal and metal oxide particles enter the brain with the respiratory tract and adhere to the cerebral cortex, it can lead to Alzheimer's disease ${ }^{3,4}$. Besides, for $\mathrm{Na}, \mathrm{K}$ and $\mathrm{Ca}$ and other heavy metal ions in the blood, the detection process are complex, expensive, not suitable for emergency or self-detection ${ }^{5}$. It is of great practical significance for taking the effective means of monitoring of heavy metal particles ${ }^{6}$. The existing monitoring technologies of particles are mainly divided into two types: sampling and non-sampling. The sampling types mainly include the weighing method, Tapered Element Oscillating Microbalance method (TEOM) and $\beta$-ray attenuation method $^{7,8}$. The non-sampling types can be divided into light transmission method, light scattering method and blackness method. In the selection of application methods, the TEOM method is the mainstream choice with high accuracy, but it requires working under constant temperature and humidity conditions and equipment is expensive and depends on im-

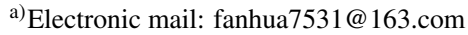

b) Electronic mail: huqiang@jihualab.com
}

ports. The $\beta$-ray attenuation method is also a very commonly used method with a low maintenance cost. However, there are shortcomings such as high cost, slow response speed, and low accuracy ${ }^{9}$. The non-sampling method uses the physical and chemical characteristics of particles to measure the concentration of particles, which is not accurate enough, but it is very suitable for on-line monitoring. A spectrophotometry system for on-line measurement of $\mathrm{Fe}, \mathrm{Mn}$ and $\mathrm{Cr}$ in environmental PM 2.5 with a time resolution of 2 hours has been proposed in the literature ${ }^{10}$. The monitor inhales and concentrates particulate matter into a slurry sample, and then measures metal concentration in the surrounding coarse particles using a microvolume flow cell (MVFC), which can be measured continuously and with high accuracy. However, this system has a long waiting time and complex operation steps, which is not conducive to large-scale promotion. A new method for PM 2.5 recognition was proposed based on a super-resolution optical neural network, which has very high accuracy, but the high-resolution scanning electron microscopy is expensive ${ }^{11}$. Refs. 12 designed a digital monitoring system that is integrated with parameters of temperature, humidity, wind speed and direction for particulate matter based on the Internet of Things (IoT), using off-the-shelf sensors as the sensing elements to construct the microsystem. There is a trade-off between high accuracy, high efficiency and low cost in the existing systems. The proposed particle measurement system uses the magnetic method, and the non-contact measurement mode not only ensures the reliability of data but also realizes online measurement. As long as the particles are located near the sensor, the sensor can immediately sense the existence of particles and trigger the change of the output voltage value, without waiting time, which improves the efficiency of data processing. The magnetic sensor based on Si material can be compatible with the CMOS process, which creates an opportunity for the sensor and AFE to be integrated into a single system on chip 
$(\mathrm{SoC})^{13}$. The volume of the instrument is small and the price is cheap, which brings hope to mass production.

Among the magnetic sensors, the Hall sensor is widely used $^{14-17}$. Hall sensor is a kind of device based on Hal1 effect $^{18,19}$. It can detect the change of the magnetic field and transform the magnetic signal into electrical signal output. In recent years, integrated Hall magnetic field sensors with high reliability, low cost, low power consumption and strong anti-interference ability have been widely used in industrial automation, instrumentation, automotive industry and consumer electronics. Hall sensor also plays an important role in ship magnetic flux leakage detection, distance detection and elevator field data acquisition. At present, many universities and research institutions have a new understanding of the special application field of Hall sensors ${ }^{20,21}$. For example, a chip based on the Hall sensor have been proposed for estimating biomedical determinations labeled with magnetic beads $^{22}$. It utilizes a short time constant that is achievable with modern sub-micron CMOS technology to perform relaxation field measurements. The system provides a feasible scheme for the measurement of magnetic particles. The SoC for rapid blood monitoring was designed using the Hall sensor, which provides a rapid and low-cost tool for disease screening ${ }^{5}$. A sandwich detection scheme based on magnetic bead was used to prefix the antibody on the surface of Hall sensor. After the antibody captured the biomarkers on the magnetic beads, the Hall sensor array based on CMOS converts the biomolecular signal into electrical signal. For distance detection or particle detection, non-destructive and non-contact detection methods can greatly improve reliability. Therefore, using a stable and economical scheme to detect and analyze the distance or magnetic particles is of great significance in protecting human health and improving the quality of life.

This paper is organized as follows: The sensor array assembly design is present in Section II, while the design of the hardware and software circuit system is given in Section III, which includes three sub-branches: analog front-end circuit (AFE), digital ADC and MCU microsystem program. The experimental results are shown in Section IV. Finally, Section V draws a conclusion.

\section{SENSOR ARRAY ASSEMBLY}

Hall devices are divided into vertical Hall device (VHD) and horizontal Hall device (HHD). The horizontal Hall device was first proposed with mature research technology and higher sensitivity. In this design, a square horizontal Hall device is selected as the sensing unit, as shown in Fig. 1(a). The Hall plate has four contact ports. The two diagonal ports are a pair. When one pair of ports is the excitation electrode, the other pair of ports is the Hall voltage output port. When detecting the magnetic field, the Hall electrode can sense the magnetic field perpendicular to the Hall plate and generate the Hall voltage only by connecting the bias electrode with a voltage source or a current source. The output voltage of the Hall sensor can be expressed as Eq. (1):

$$
V_{H}=R_{H} \frac{I \cdot B}{t}
$$

where $I$ is the bias current, $t$ is the thickness of the doped ion region, $B$ is magnetic field, and $R_{H}$ is the Hall coefficient. The depth of the active region of Hall device fabricated by the CMOS process is relatively shallow, and the doping concentration in the region is Gaussian distribution. According to the above formula, the smaller $t$ is, the greater Hall voltage is, so the CMOS process is very suitable for horizontal Hall sensor. When the DC voltage source is selected as the excitation source, the voltage related sensitivity of the sensor is obtained in Eq. (2):

$$
S_{V}=\frac{V_{H}}{V_{D D} B}
$$

where $V_{D D}$ is the supply voltage. The working principle of the Hall sensor includes magnetic field and electric field, so it is necessary to build a suitable magnetoconductance model to realize the coupling of the magnetic field and conductivity. In the simulation, we set the conductivity to tensor mode, the magnetic field is included, which can make the magnetic field change and affect the conductivity of the device at the same time, so as to simulate the Hall effect ${ }^{18}$.

The inherent magnetic field of most of the metal particles in the air is too weak to cause significant changes in the output of the sensor. How to enhance the magnetic field around the particles to make the sensor produce enough output becomes the key problem to be solved in this design. The proposed design puts forward an innovative solution to the problems mentioned above: depositing a layer of a metal coil on the back of the chip. After the metal coil is electrified, current will be generated, and then a magnetic field will be generated according to the electromagnetic effect, thus the environmental magnetic field will be generated to magnetize the metal particles. The magnetized metal particles will be adsorbed on the chip surface and cause the output voltage of the Hall sensor to change. For the detection of particulate matter, the Hall array is adopted in this paper.

1) If a single large Hall plate is used, the magnetic field generated by the coil is not uniform on the Hall plate, which leads to different magnetic fields reflected by the voltage measured at different positions, thus it is impossible to judge the situation of particles. The accuracy and uniformity of this method need to be improved;

2) If the small Hall plate is used, the larger size particles can not be measured, and the upper limit of the number of particles that can be measured at one time is reduced, which is greatly affected by random and accidental factors, which will lead to the decrease of the credibility of the measurement results.

Considering the above two aspects, the design adopts the form of Hall plate array and the Hall plate with a smal1 area, which approximately considers that the magnetic field in the plate is uniform ${ }^{23}$. The number of particles in a wide 


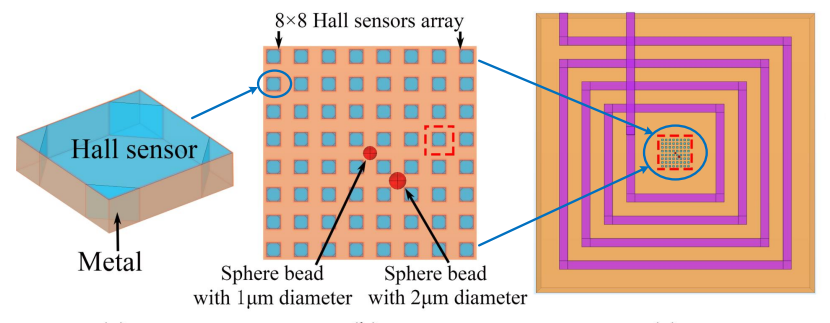

(a)

(c)
FIG. 1. Schematic design of Hall array and back coil design. (a) single square Hall sensor; (b) $8 \times 8$ Hall array composed of 64 Hall sensors; (c) The diagram of metal coil placed on the back of the chip.

range can be measured by using multi-chip array measurement, which has good performance against random and accidental factors. As shown in Fig. 1, the designs of Hall array and back coil by COMSOL Multiphysics software are shown.

In the design, the Hall array is divided into two group$s$, which differ only in the presence or absence of particulate matter. One group of sensor array with particles is used to measure the magnetic field changes when particles exist, while the other group is used to record the interference of geomagnetic and other environmental magnetic fields. The design parameters and simulation environment of sensors and metal coils in the two groups are the same. The difference in the output voltage of the sensors at the corresponding positions on the two Hall arrays is the voltage change that represents the magnetic field variation caused by the particles.

When the material is magnetized by the external magnetic field $B_{0}$, the additional magnetic field $B^{\prime}$ is generated, and the total magnetic field $B=B_{0}+B^{\prime}$. Materials are divided into three parts: paramagnetic materials, diamagnetic materials and ferromagnetic materials. The additional magnetic field produced by paramagnetic and diamagnetic materials is very small, which is generally not considered. Therefore, this project is focused on ferromagnetic materials such as Fe, Co, $\mathrm{Ni}$, etc. are mainly considered in this project because of their high permeability. Besides, the surface oxidation of materials and alloy problems need to be considered, which need to be further verified by experiments.

\section{CIRCUIT DESIGN}

\section{A. Design of Analog Front End Circuit}

The analog front-end (AFE) circuit includes a spinning current circuit, differential amplifier, common-mode feedback network and two-stage chopper circuit. As shown in Fig. 2, it is the frame diagram of AFE. Due to the interference of nonideal factors such as process variation, asymmetric structure or inhomogeneous doping, some inherent offsets exist in Hall sensors, which leads to the output Hall voltage not being zero when the applied magnetic field is zero ${ }^{24,25}$. The spinning current circuit can be used to eliminate the misalignment error and flicker noise of the Hall sensor dynamically. As long as the clock frequency is set properly, the change of the magnetic field is much slower than that of the clock frequency. The spinning current circuit complements the periodic change of clock to control whether the MOS transistor is on, thus changing the power supply direction and output port. The output Hall voltage changes periodically with the phase change of bias voltage, but the offset voltage is inherent. This offset voltage can be eliminated by using a spinning current circuit ${ }^{26,27}$. The result of subtracting the voltage measurements between different phases is twice the Hall voltage. Due to the performance requirements of the ADC module, Hall voltage must be amplified to a certain range before the analog-to-digital conversion can be carried out ${ }^{28}$. The bias circuit is composed of a bandgap reference and a current mirror to provide various bias voltage values required by the amplifier. But the amplifier circuit will introduce additional offset and noise, so we need a two-stage chopper circuit to modulate the offset and noise introduced by the amplifier circuit to high frequency, which is convenient for the subsequent low-pass filter circuit to filter out the high-frequency noise. Due to the process problems, the static operating point of the fully differential amplifier circuit is often unstable, so the common mode feedback circuit is needed to stabilize the static operating point so that all the transistors of the amplifier can work normally in the saturation region. As a transition module between the analog front-end circuit and ADC, buffer plays an important role in increasing load capacity. The circuit model of Hall sensor adopts the equivalent Wheatstone bridge structure of resistance divider type in Refs. 5.

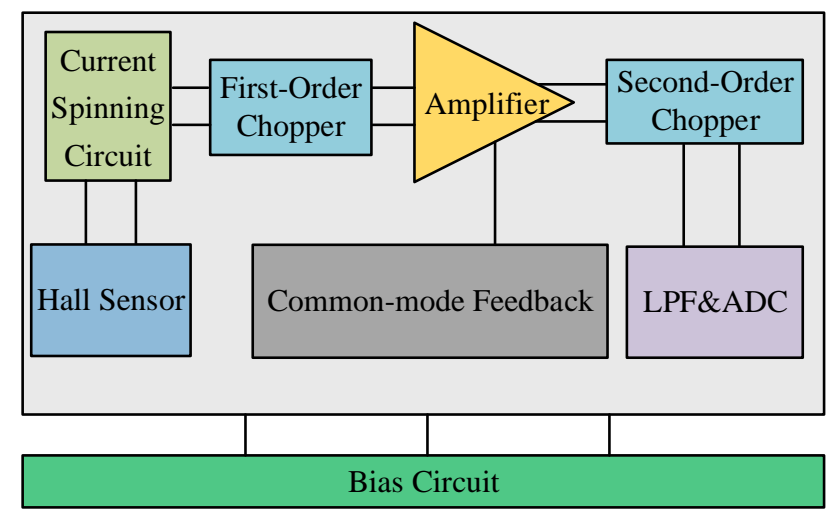

FIG. 2. The composition diagram of analog front end circuit.

\section{B. Design of Digital ADC}

In the case of high-speed $\mathrm{ADC}$, this paper proposes to use digital PWM delta-sigma ADC to convert the analog signal into a digital signal, which improves the ability of anti-noise and anti-distortion and reduces the area and power consumption ${ }^{29-31}$. In this circuit, "dout" is 12 bit ADC output and "rcctrl" is used to output PWM voltage and connect with the input of the RC network. The "rcout" is the filtered analog 
voltage output from RC network and connected to the negative end of the comparator. If the comparator output is logic " 1 ", the analog input is higher than the output voltage of the RC circuit. The logic " 1 " is sampled by the trigger and feedback to the RC circuit, which makes the output voltage of the RC circuit rise, that is, the negative terminal voltage of the comparator rises. If the output of the comparator is logic " 0 ", then the feedback signal will be logic " 0 " and the the RC output voltage will be reduced, that is, the negative terminal voltage of the comparator will drop. Through this simple feedback mechanism, the output value of the RC circuit will "track" the frequency of analog input. The time constant $\tau=\mathrm{RC}$ should be large enough to filter out the high-frequency part of PWM, but not so large as to suppress the response time ${ }^{32}$. In this design, the capacitance value is $561 \mathrm{pF}$, the resistance value is $60 \mathrm{k} \Omega$, that is, $\tau=3.366 \mu \mathrm{s}$, and the clock is $50 \mathrm{MHz}$. In the simulation of the digital module of the analog-to-digital converter, eight accumulators are used to delay the sampling period of ADC one by one to increase the sampling frequency.

\section{MCU Program}

A micro-system needs a master chip to complete a series of operations and control. After analog front-end circuit processing and ADC sampling, the conversion process of Hall voltage and magnetic field strength needs to be completed by $\mathrm{MCU}^{33}$. At the same time, the detection and data display of magnetic field information also need to be judged by the master chip, control the movement of the stepper motor, realize the communication between master chip and PC, and complete the real-time display of LCD1602. Due to the advantages of high precision, low power consumption and strong expansibility, the STM32F103ZET6 chip is selected as the main control chip in this design. As shown in Fig. 3, it is the composition and structure framework of the whole microsystem. In the design, the power supply voltage of the stepper motor drive module, Hall sensor module and STM32 series MCU module are $12 \mathrm{~V}, 5 \mathrm{~V}$ and $3.3 \mathrm{~V}$ respectively. DC stabilized power supply circuit is generally composed of transformer module, half-wave rectifier circuit, LC or LC $\Pi$ or RC $\Pi$ filter circuit and voltage stabilizing circuit. After $220 \mathrm{~V}$ AC voltage passes through a single-phase bridge rectification circuit and then passes through an LC filter, a DC power supply can be obtained. The LM2596 switching regulator is used in this microsystem to change $12 \mathrm{~V}$ DC input into $5 \mathrm{~V}$ DC output, and then realizes the conversion of $5 \mathrm{~V}$ DC voltage input to 3.3 V DC voltage output through the AMS1117 linear regulator chip.

The software design of the magnetic field detection system consists of two parts: the upper computer operation interface and the lower computer control program. The main functional requirements of the upper computer are to complete the serial communication protocol setting, the motion parameter setting of the lower computer, the control of the motion direction, the data storage and real-time display of the magnetic field size and position information. For different objects to be measured and different measurement requirements, the scanning speed, step size, sampling point and other parameters of the corresponding sensor are different. The setting of the upper computer needs to realize the detection automation to the greatest extent. Due to the limited length of the mechanical slide rod, it is necessary to set the endpoint of motion. After the sensor reaches the endpoint, the motion mode of the stepper motor changes from forward to reverse and returns to the starting point again. As shown in Fig. 4, it shows the program design flow of the upper computer control software.

The controller of the lower computer is the main control chip, STM32F103 series microcontroller. The design of the lower computer is used to realize the motion control function of the stepping motor, the communication between the upper computer and the lower computer, the data acquisition and the realization of the motion scanning algorithm. In the design process of the algorithm, the coordination between the function realization of each module and the three-dimensional mechanical motion platform should be considered. As shown in Fig. 5 is the flow chart of the lower computer control program.

\section{EXPERIMENT AND RESULTS}

\section{A. Sensor Array Assembly}

The Ansoft Maxwell 16.0 is professional electromagnetic simulation software, which can easily simulate the magnetic field changes caused by particles. As shown in Fig. 6, it is the simulation to compare the change of magnetic field with or without a nickel particle. On the left is the magnetic field generated by the electromagnet, and on the right is the magnetic field change when a nickel particle with a diameter of 2.5 $\mu \mathrm{m}$ is placed in the same magnetic field environment. It can be seen that there is an obvious magnetic field enhancement effect near the nickel particle.

The Hall sensor is a kind of device based on the Hall effect, which is the key to realize magnetoelectric conversion. However, Maxwell software can not simulate the Hall effect, because the phenomenon involves the coupling problem of electric field and magnetic field. The COMSOL Multiphysics is a multi-physical fields simulation software, which can realize

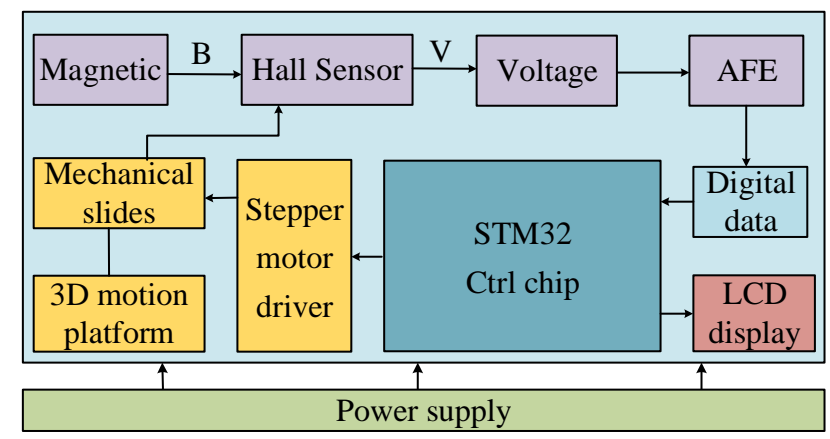

FIG. 3. Structural framework of micro system based on Hall sensor. 
the coupling and modeling of multiple physical fields such as electric field, magnetic field, acoustics, thermodynamics and material chemistry. In this software, we can easily establish the relationship between electromagnetic fields by introducing anisotropic conductivity. Besides, in order to avoid simulation error of single software, multi-software simulation is also necessary. In this paper, Maxwell software simulates the magnetic field changes caused by particles. The COMSOL Multiphysics software is to put the particles into the Hall array and obtain the output voltage variation. The Hall effect can realize the conversion between magnetic signal and electrical signal. The magnetic field change caused by the existence of particles is converted into voltage change by the Hall array.

The software version used in the simulation is COMSOL multiphysics 5.5. In this model, a $2 \times 2 \mu \mathrm{m}$ square Hall device with a thickness of $0.5 \mu \mathrm{m}$ is selected as the sensing element.

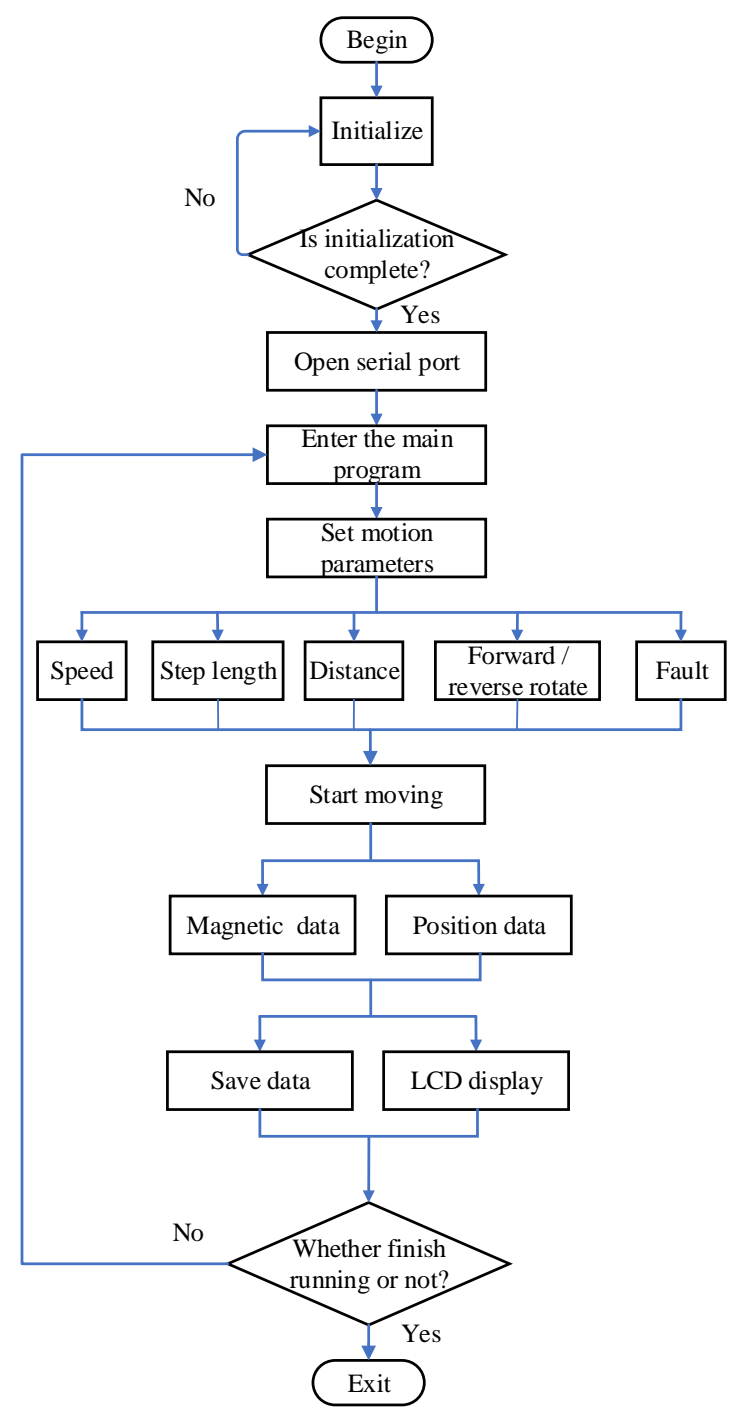

FIG. 4. The flow chart of the user-interface software.
If the size of the Hall device is too large, when the magnetic field enhancement area produced by the particles is equal to the size of sensor, the Hall voltage may have little or almost no change because the perceived area of the Hall device is too small. Therefore, the size of the Hall device had better to be equal to the size of the particles. Only in this way can the Hall sensor output voltage be significantly enhanced. To be compatible with the CMOS process and realize on-chip integration, the sensor uses Si material with $\mathrm{N}$ doping, which the concentration is $10^{17} \mathrm{~cm}^{-3}$. The triangle area of the four corners of the square is selected as metal to form an ohmic contact with the semiconductor. The power supply voltage of a single Hall device is $5 \mathrm{~V}$, and the measured voltage related sensitivity is about $0.11 \mathrm{~V} / \mathrm{V} / \mathrm{T}$. The distance between Hall devices is $2 \mu \mathrm{m}$. To obtain a larger Hall voltage as much as possible, the parameter setting of the metal coil needs to meet the condition that the coil can produce a magnetic field that is perpendicular to the Hall device, and the magnetic field is at

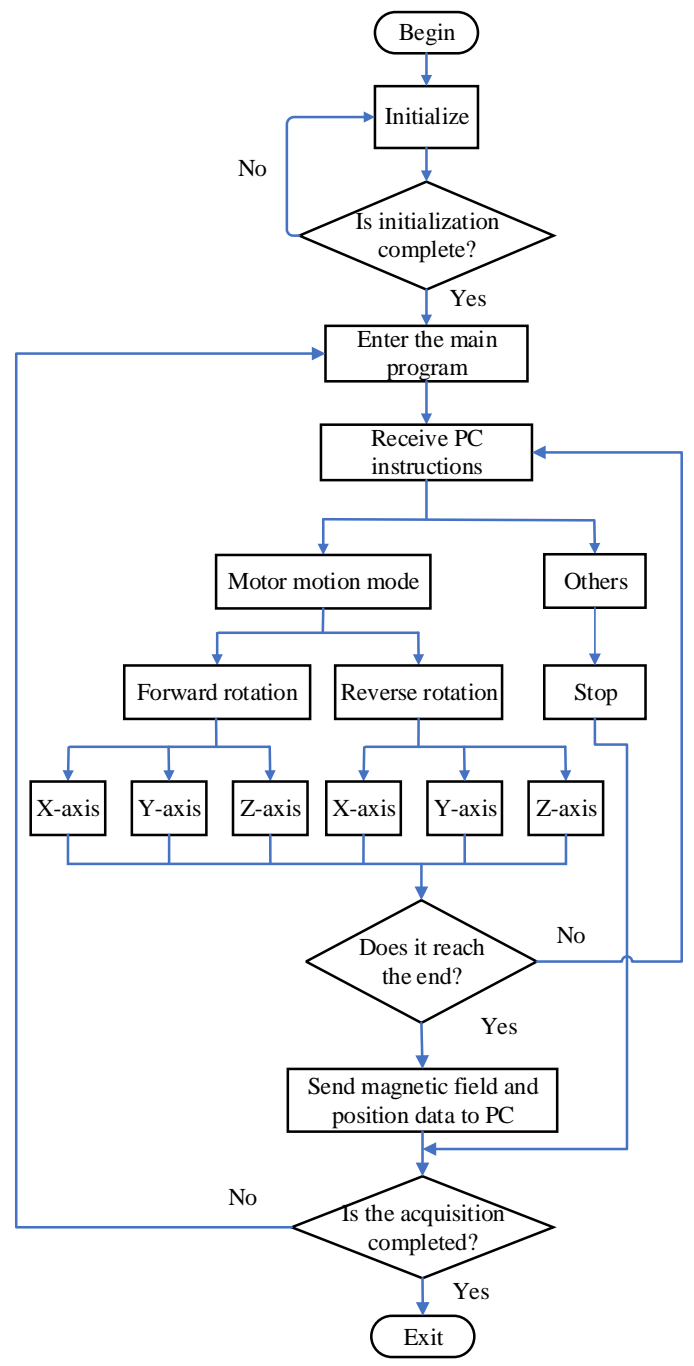

FIG. 5. The flow chart of MCU control program. 
least one order of magnitude larger than the geomagnetic field. After parametric scanning in the COMSOL multiphysics, we selected the width and the height of the coil cross-section to be $9 \mu \mathrm{m}$ and $4.5 \mu \mathrm{m}$. As shown in Fig. 7, it is the 3D COMSOL technologically constructive model. This software has the function of mesh calibration and pre-defined, which can automatically adjust and optimize the simulation. The userdefined mesh mode is used in the whole model, which can make the simulation more accurate. Following the principle that the smaller the volume, the more precise the mesh, we selected the predefined mesh sizes of particles and sensors to be "extra fine" and "finer". The volume of the metal coil is the largest, and its mesh can be set coarser. To reduce the calculation time, the minimum mesh cell of the metal coil is defined to be consistent with the size of the Hall devices. Therefore, the minimum mesh cell parameters of particles, sensors and metal coil are $0.45 \mu \mathrm{m}, 1.2 \mu \mathrm{m}$ and $2 \mu \mathrm{m}$ respectively.

The distance between the particles and the sensors should not be too large, because the magnetic field generated by the particles is relatively small. If the distance is too far, the sensor will not detect the existence of particles. Due to the process of chip manufacturing and packaging, the distance between particles and the Hall array can not be too close. It should be noted that the output voltage of Hall sensor will fluctuate due to the interference of the geomagnetic field and the presence of magnetic field noise generated by nearby power equipment. In this design, the distance between the particle and the sensor is set to $3 \mu \mathrm{m}$, which ensures that the sensor array can produce as much output voltage as possible in the measurable range ${ }^{22}$. The relative permeability of the particles is 4000. During simulation verification, when applied $30 \mathrm{~mA}$ bias current to the proposed metal coil, it can gener-

(a)

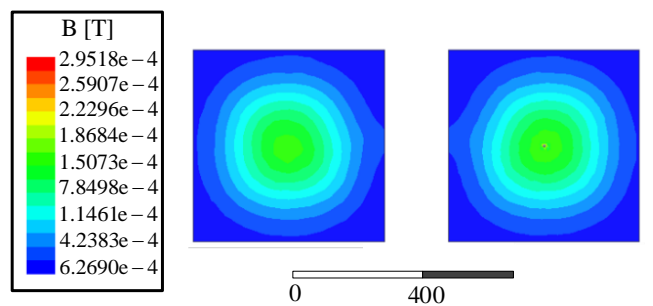

(b)

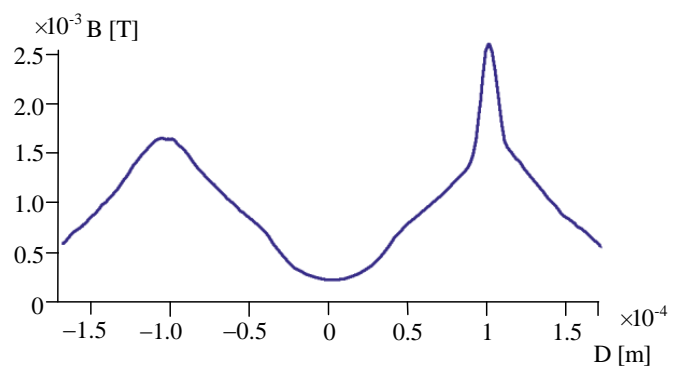

FIG. 6. The existence of particles causes the enhancement of environmental magnetic field. (a) The magnetic field distribution diagram; (b) the magnetic field size without particles (left) and the magnetic field size with particles (right).

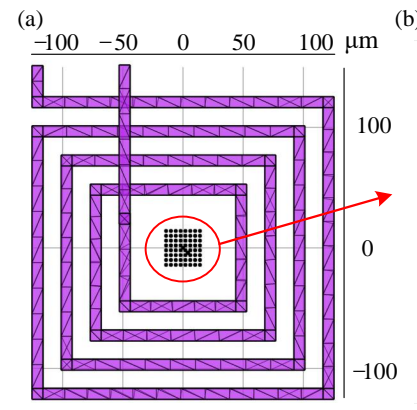

(c)

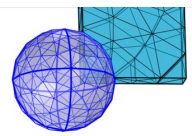

b)
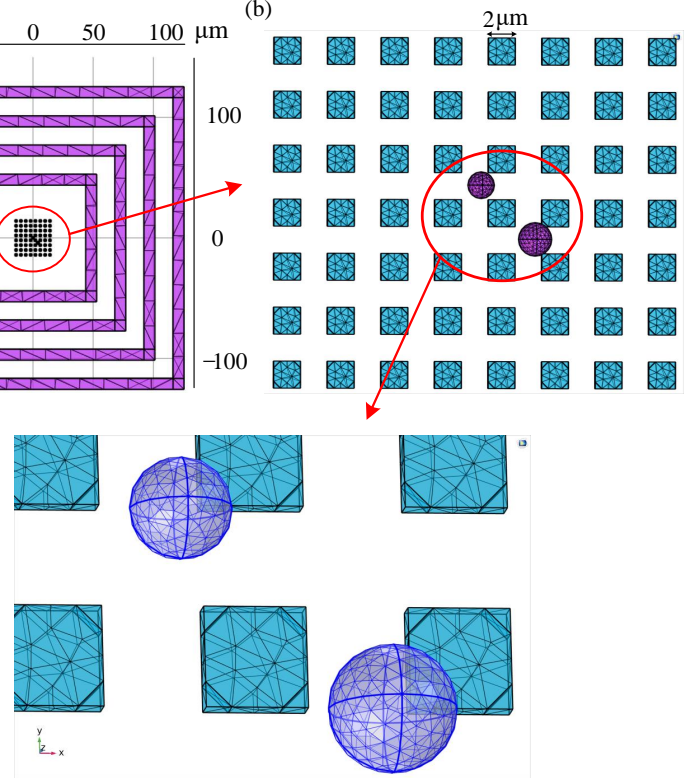

FIG. 7. The 3D COMSOL meshed models of Hall array, metal coil and particles. (a) The mesh of metal coil; (b) The mesh of Hall array; (c) The mesh of particles.

ate about $10^{-4}-10^{-3}$ Tesla magnetic field and microvolt level Hall voltage.

The diameter of particle $\mathrm{A}$ is $1 \mu \mathrm{m}$, and that of particle $\mathrm{B}$ is $2 \mu \mathrm{m}$ in Fig. 8. In the simulation process, B keep still and gradually reduce the distance between the two particles. First of all, after the particles are magnetized, an additional magnetic field will be generated in space, this magnetic field intensity is about $1-20 \mu \mathrm{T}$; secondly, the additional magnetic field generated by the magnetized particles will be detected by the nearby Hall sensors, which will convert the magnetic variation into the change of output voltage. The voltage change is about $1-4 \mu \mathrm{V}$. The output voltage signal is discrete data, so the stereogram below Fig. 8 is used to represent the voltage difference between the two Hall arrays, that is, the voltage change caused by particles. In Fig. 8(a), the sensor voltage variation near particle $\mathrm{A}$ is $1 \mu \mathrm{V}$, and the sensor voltage change value near particle $\mathrm{B}$ is $2 \mu \mathrm{V}$. From Fig. 8(a) to Fig. 8(c), particle A gradually approaches particle B. because the distance between the two particles is always large and the interaction between particles is weak, the sensor output near particle $\mathrm{B}$ remains unchanged. When two particles are close to each other, as shown in Fig. 8(d), particle B is affected by particle $A$, and the output variation of the Hall sensor nearby is about $3-4 \mu \mathrm{V}$. It can be seen from the simulation result$\mathrm{s}$ that the larger particle size causes greater voltage variation. For the same Hall sensor, when two particles are close to each other, the Hall voltage is significantly higher than that when only one particle exists.

The application scenario of this microsystem is that the air complexity is relatively light. In this case, the particle size is very small, and the distance between particles is generally much larger than their size. In this case, the interaction be- 


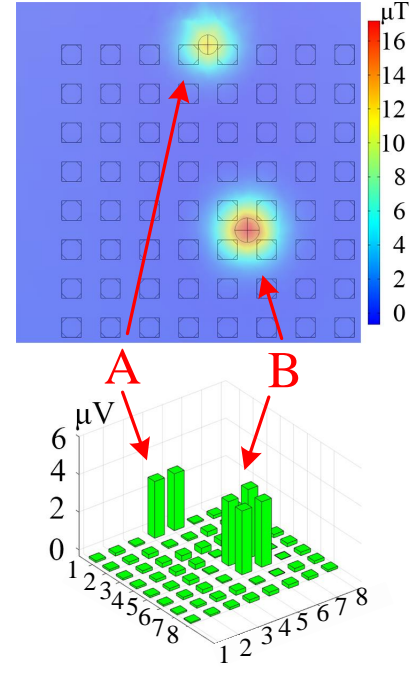

(a)
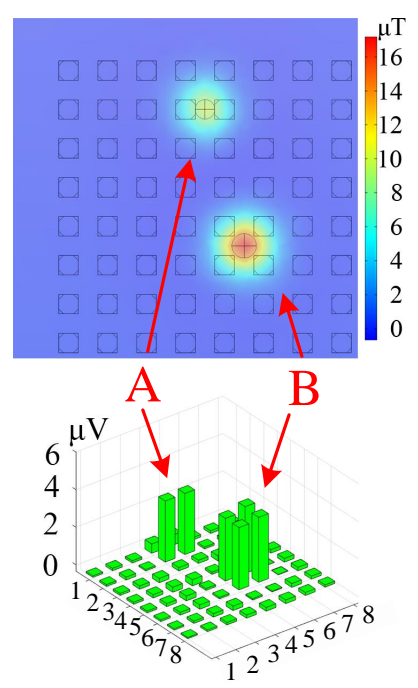

(b)
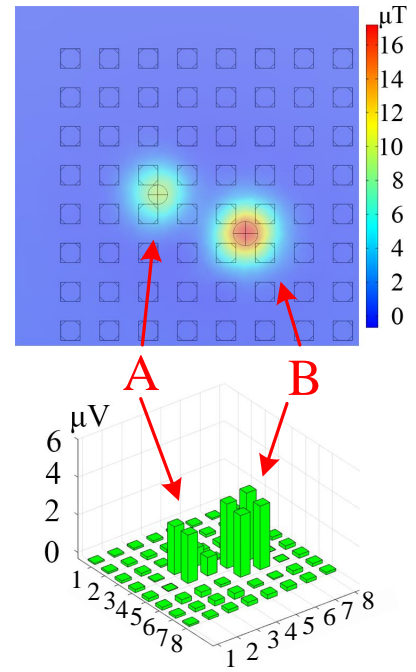

(c)
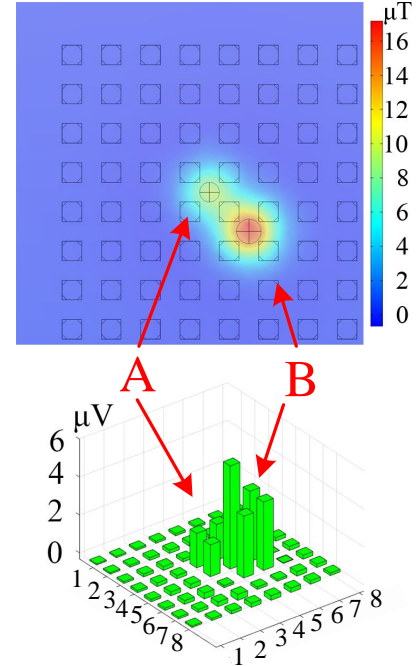

(d)

FIG. 8. The voltage output of the hall array varies with the position of the particles (The particle with a diameter of $2 \mu \mathrm{m}$ are fixed at coordinates $(5,-5))$. (a) Particles with diameter of $1 \mu \mathrm{m}$ are located at coordinates $(0,15)$; (b) particles with diameter of $1 \mu \mathrm{m}$ are located at coordinates $(0,10)$; (c) particles with diameter of $1 \mu \mathrm{m}$ are located at coordinates $(-5,0)$; (d) particles with diameter of $1 \mu \mathrm{m}$ are located at coordinates $(0,0)$.

tween particles can be ignored. The experimental results also verify this conclusion. In Fig. 8(a) and Fig. 8(b), the distance between the two particles is about $20 \mu \mathrm{m}$ and $10 \mu \mathrm{m}$. As the distance decreases, the output voltage of the Hall sensor near the particles below does not change.

The minimum voltage resolution of the analog front-end circuit in this design is $1 \mu \mathrm{V}$ for the input signal, so the $1 \mu \mathrm{V}$ voltage value is used as the dividing line in the sensor simulation design. In the simulation design of COMSOL, metal particles are artificially added to simulate the metal particles in the air. The results show that when the particle size is $1 \mu \mathrm{m}$ or less, the output Hall voltage is as low as $1 \mu \mathrm{V}$. Therefore, we consider that the minimum diameter of detectable magnetic particles in the proposed system is $2 \mu \mathrm{m}$.

The material used in this model is $\mathrm{Si}$. When the bias voltage of Hall devices is $5 \mathrm{~V}$, the output of 64 Hall devices is several microvolts. The GaAs material has a wide bandgap and excellent temperature characteristics, which is suitable for the design of high sensitivity Hall sensors. In theory, the output of Hall devices can be increased by $5-6$ times by changing into GaAs material, so that the Hall voltage can exceed 10 $\mu \mathrm{V}$. However, due to the incompatibility between GaAs material and the CMOS process, the sensor can only be integrated off-chip, which will greatly increase the complexity and cost of the design. Hall sensor based on Si material is compatible with the CMOS process, and has a long history, the technology is mature, so in consideration of reliability and cost, this model adopts Si material.

\section{B. Circuit Performance}

The amplifier circuit is mainly composed of a differential amplifier circuit, common mode feedback circuit and bias circuit. The amplifier is a single-stage full differential amplifier with common mode feedback. The main body of the amplifier is a folded cascode structure to increase the swing of the output voltage. According to the characteristics of digital ADC, the amplification requirements of the analog front-end circuit can be reduced. The simulation results of the main performance index of the circuit in Cadence are shown in Fig. 9(a). The experimental results show that the small-signal gain is $40.869 \mathrm{~dB}$, the phase margin (PM) is $68.109^{\circ}$ and the bandwidth is $3.04 \mathrm{GHz}$. The common-mode rejection ratio (CM$\mathrm{RR}$ ) of the circuit is $64.349 \mathrm{~dB}$. It can be seen that the amplifier is very stable. It is not necessary to worry that the input change of the sensor will affect the amplifier magnification. The results show that the output noise of the analog frontend circuit is stable at $25.528 \mathrm{pV}^{2} / \mathrm{Hz}$ when the frequency is greater than $100 \mathrm{~Hz}$ as shown in Fig. 9(b). The overall power consumption of the analog front-end circuit is $2.16 \mathrm{~mW}$, which is greatly superior to the result $(4.2 \mathrm{~mW})$ of the analog readout circuit of the Hall sensor system designed by the same process in Refs. 34. In this paper, the sampling frequency of the proposed digital ADC is $12 \mathrm{bit} / 1.31 \mathrm{~ms}$. After the simulation, the results of ADC are shown in Fig. 9(c). The spurious free dynamic range (SFDR) is $90.0812 \mathrm{~dB}$, signal to noise and distortion ratio (SNDR) is $70.6142 \mathrm{~dB}$ and the effective number of bits (ENOB) is 11.4448-bit. Table I shows the summary of readout circuit performance.

Because STM32 has advanced clock management, excel- 


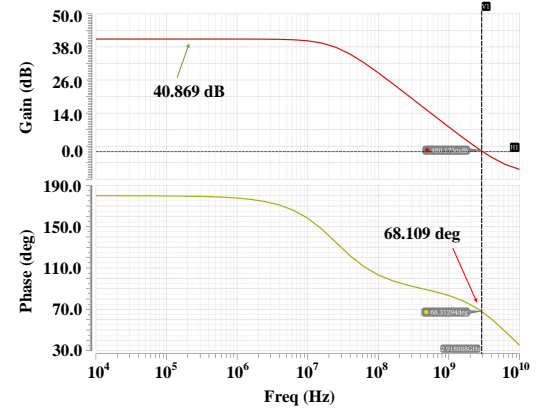

(a)

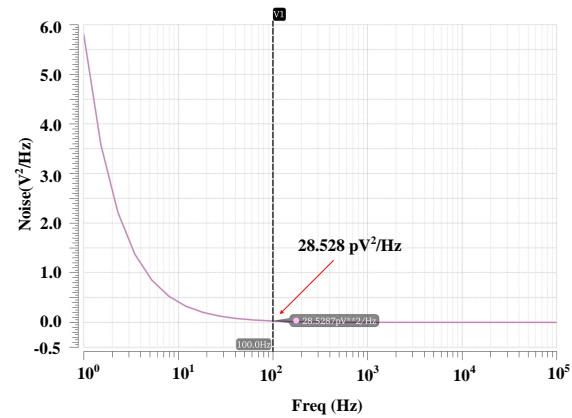

(b)

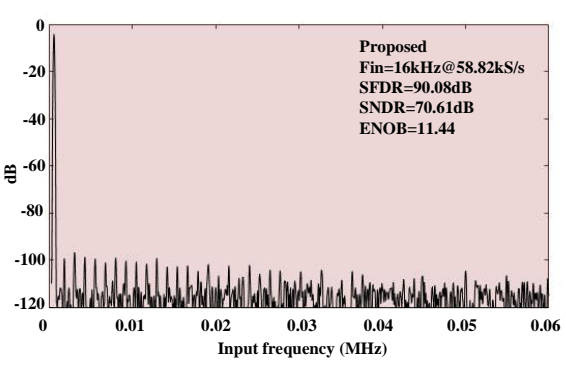

(c)

FIG. 9. Simulation results of analog front end circuit. (a) Amplitude frequency characteristic of operational amplifier; (b) Noise response curves of operational amplifier; (c) The output spectrum results of ADC by FFT.

TABLE I. Summary of full readout circuit performance.

\begin{tabular}{l|l}
\hline \hline Technology & $0.18 \mu \mathrm{m}$ CMOS \\
\hline Magnetic Particle Size & $2 \mu \mathrm{m}$ (Diameter) \\
\hline Sensitivity & $0.28 / \mathrm{T}$ (Sensor element) \\
\hline Power & $2.16 \mathrm{~mW}$ (AFE) \\
\hline Amplifier Architeture & Differential Difference \\
\hline Gain (Av) & $40.869 \mathrm{~dB}$ (Amplifier) \\
\hline Phase margin (PM) & $68.109^{\circ}$ (Amplifier) \\
\hline Bandwidth & $3.04 \mathrm{GHz}$ (Amplifier) \\
\hline CMRR & $64.349 \mathrm{~dB}$ (Amplifier) \\
\hline SFDR & $90.0812 \mathrm{~dB}$ (ADC) \\
\hline SNDR & $70.6142 \mathrm{~dB}$ (ADC) \\
\hline ENOB & $11.4448 \mathrm{bits}$ (ADC) \\
\hline \hline
\end{tabular}

lent power supply and high-performance ADC with fast sampling, we can not only improve the real-time performance of data acquisition but also increase the reliability and accuracy of data. With necessary experimental verification, the realtime data displayed on LCD1602 screen when the microsystem works normally. The interval between each refresh of LCD is 1.34 seconds, which is convenient for users to observe and does not affect the continuous sampling of data. After the software simulation and debugging, the whole module works normally. The signal detected by the Hall sensor can be collected online in real time. The expected data acquisition function is achieved, and the collected value can be displayed in LCD1602 in real time. The reasonable design of the circuit can effectively reduce the overall cost and power consumption of the module, which is a good choice for particle detection and distance measurement. With the development of the integrated circuit industry and the efforts of researchers, the microsystem based on Hall sensor will be integrated, easy to operate and portable, providing new guarantee for the better life of human beings.

\section{CONCLUSION}

This paper proposes a solution to detect particulate matter using magnetism. This method can achieve non-contact measurement, convenient, efficient and high accuracy. The proposed Hall array greatly improves the measurement sensitivity, and the simulation results show that the detectable particle diameter is as low as $2 \mu \mathrm{m}$. The main control chip adopts the smallest system board of STM32F103 series microcontroller supported by $3.3 \mathrm{~V}$ power supply voltage, which has the advantages of high performance, low power consumption and strong expansibility, and the design of voltage stabilizing module and serial communication module makes the stable transmission of magnetic data. The microsystem has completed the cooperative operation of software and hardware and can realize the scanning and detection of space magnetic field signal. During the scanning process, the magnetic field information can be displayed on the upper computer and LCD1602 in real time. The experimental results show that the detection system works well and the data is correct, which can effectively collect the space magnetic field data. The whole detection system is stable and efficient, and the measurement results are intuitive and accurate. Nowadays, people pay more attention to health and have a higher pursuit of the quality of life. The design of this paper has a strong application value for the realtime rapid detection of heavy metal particles in the air and blood analyzer. It is hoped that this design can provide some new ideas and new directions for society to solve the problems in these two application fields.

\section{ACKNOLEDGEMENT}

The work of Hua Fan was supported by the National Natural Science Foundation of China (NSFC) under Grant 61771111, supported by Sichuan Provincial Science and Technology Important Projects under Grant 19ZDYF2863, Supported by the Open Foundation of State Key Laboratory of Electronic Thin Films and Integrated Devices under Grant KFJJ202006.

The work of Quanyuan Feng was supported by the Ma- 
jor Project of the National Natural Science Foundation of China (NSFC) uner grant 62090012, supported by the National Natural Science Foundation of China (NSFC) under Grant 61531016, supported by Sichuan Provincial Science and Technology Important Projects under Grant 2018GZ0139, 2018ZDZX0148 and 2018GZDZX0001.

\section{DATA AVAILABILITY STATEMENT}

The data that support the findings of this study are available from the corresponding author upon reasonable request.

${ }^{1}$ J.-H. Yi, X.-F. Wu, L.-Y. Wang, J. Lei, and J. Han, "Mechanisms and effects of pm 2.5 on diseases of respiratory system: Latest findings," Journal of Xi' an Jiaotong University (Medical Sciences) 40, 167-172 (2019).

${ }^{2}$ T. Tzeng, C. Kuo, S. Wang, P. Huang, Y. Huang, W. Hsieh, Y. Huang, P. Kuo, S. Yu, S. Lee, Y. J. Tseng, W. Tian, and S. Lu, "A portable micro gas chromatography system for lung cancer associated volatile organic compound detection," IEEE Journal of Solid-State Circuits 51, 259-272 (2016).

${ }^{3}$ B. Maher, I. Ahmed, V. Karloukovski, D. MacLaren, P. Foulds, D. Allsop, D. Mann, R. Torres-Jardón, and L. Calderon-Garciduenas, "Magnetite pollution nanoparticles in the human brain," Proceedings of the National Academy of Sciences 113, 10797-10801 (2016).

${ }^{4}$ A. Barbara, Maher, A. M. Imad, Ahmed, Vassil, Karloukovski, A. Donald, MacLaren, G. Penelope, and F. and, "Magnetite pollution nanoparticles in the human brain." Proceedings of the National Academy of Sciences of the United States of America (2016).

${ }^{5}$ P. Kuo, J. Kuo, H. Hsueh, J. Hsieh, Y. Huang, T. Wang, Y. Lin, C. Lin, Y. Yang, and S. Lu, "A smart cmos assay soc for rapid blood screening test of risk prediction," IEEE Transactions on Biomedical Circuits and Systems 9, 790-800 (2015).

${ }^{6}$ F. Guevara Luna, M. Guevara-Luna, and N. Rojas, "Spatial-temporal assessment and mapping of the air quality and noise pollution in a sub-area local environment inside the center of a latin american megacity: Universidad nacional de colombia - bogotá campus," Asian Journal of Atmospheric Environment 12, 193-204 (2018).

${ }^{7}$ S. Pattanaik, F. Huggins, and G. Huffman, "The variability in iron speciation in size fractionated residual oil fly ash particulate matter (rofa pm)," Science of The Total Environment 562, 898-905 (2016).

${ }^{8}$ X. Zhang, L. Zhang, S. Gong, L. Tian, Y. Zhang, L. Yang, and Z. Jia, "Design of pm 2.5 air quality detection system based on median value average filtering algorithm and oled screen," IOP Conference Series: Earth and Environmental Science 450, 012013 (2020).

${ }^{9}$ C.-P. Su, X. Peng, X. Huang, L.-W. Zeng, L. Cao, M.-X. Tang, Y. Chen, B. Zhu, Y. Wang, and L. He, "Development and application of a mass closure pm 2.5 composition online monitoring system," Atmospheric Measurement Techniques 13, 5407-5422 (2020).

${ }^{10}$ D. Wang, M. H. Sowlat, and J. Schauer, "Development and evaluation of a novel monitor for online measurement of iron, manganese, and chromium in ambient particulate matter (pm)," Science of The Total Environment $\mathbf{5 6 5}$, 123-131 (2016).

${ }^{11}$ A. Kokoulin and R. Kokoulin, "Multiscale optical pm 2.5 particles recognition and sorting system in dust probes," (2020) pp. 188-193.

${ }^{12}$ R. Simanullang, S. Soekirno, and H. Larassari, "Design and analysis of air quality monitoring system $\mathrm{pm} 10$ and pm 2.5 integrated with weather parameters (a case study on margonda raya street depok)," Journal of Physics: Conference Series 1528, 012053 (2020).

${ }^{13}$ P. Ciccarella, M. Carminati, M. Sampietro, and G. Ferrari, "Multichannel $65 \mathrm{zf}$ rms resolution cmos monolithic capacitive sensor for counting single micrometer-sized airborne particles on chip," IEEE Journal of Solid-State Circuits 51, 2545-2553 (2016).
${ }^{14}$ V. Nabaei, R. Chandrawati, and H. Heidari, "Magnetic biosensors: Modelling and simulation," Biosensors and Bioelectronics 103, 69-86 (2018).

${ }^{15} \mathrm{H}$. Fan, S. Li, V. Nabaei, Q. Feng, and H. Heidari, "Modeling of threeaxis hall effect sensors based on integrated magnetic concentrator," IEEE Sensors Journal 20, 9919-9927 (2020).

${ }^{16}$ S. Abersfelder, A. Meyer, A. Heyder, M. Herzog, J. Franke, and M. Brela, "Crack detection in pm-rotors using magnetic field measurement and data processing," (2017) pp. 1-6.

${ }^{17}$ H. Heidari, E. Bonizzoni, U. Gatti, F. Maloberti, and R. Dahiya, "Cmos vertical hall magnetic sensors on flexible substrate," IEEE Sensors Journal 16, 8736-8743 (2016).

${ }^{18}$ B. Liu, Y. Sun, Y. Ding, P. Cao, A. Liu, S. Y. Ong, M. Tiong, G. Cheng, M. N. Islam, R. Jain, T. L. Tan, E. Quek, and E. Toh, "Low-power and high-sensitivity system-on-chip hall effect sensor," in 2017 IEEE SENSORS (2017) pp. 1-3.

${ }^{19} \mathrm{C}$. Lee and T. Chien, "Design and fabrication of micro-hall-effect sensors," in 2018 12th International Conference on Sensing Technology (ICST) (2018) pp. 96-101.

${ }^{20} \mathrm{H}$. Heidari and V. Nabaei, Magnetic Sensors for Biomedical Applications (2019).

${ }^{21}$ K.-C. Cheng, C.-H. Tseng, and L. M. Hildemann, "Using indoor positioning and mobile sensing for spatial exposure and environmental characterizations: Pilot demonstration of pm 2.5 mapping," Environmental Science \& Technology Letters 6, 153-158 (2019).

${ }^{22}$ P. P. Liu, K. Skucha, Y. Duan, M. Megens, J. Kim, I. I. Izyumin, S. Gambini, and B. Boser, "Magnetic relaxation detector for microbead labels," IEEE Journal of Solid-State Circuits 47, 1056-1064 (2012).

${ }^{23}$ S. Gambini, K. Skucha, P. P. Liu, J. Kim, and R. Krigel, "A 10 kpixel cmos hall sensor array with baseline suppression and parallel readout for immunoassays," IEEE Journal of Solid-State Circuits 48, 302-317 (2013).

${ }^{24} \mathrm{H}$. Heidari, E. Bonizzoni, U. Gatti, and F. Maloberti, "A cmos currentmode magnetic hall sensor with integrated front-end," IEEE Transactions on Circuits and Systems I: Regular Papers 62, 1270-1278 (2015).

${ }^{25}$ D. Issadore, Y. I. Park, H. Shao, C. Min, K. Lee, M. Liong, R. Weissleder, and H. Lee, "Magnetic sensing technology for molecular analyses," Lab on A Chip 14, 2385-2397 (2014).

${ }^{26}$ V. Mosser, N. Matringe, and Y. Haddab, "A spinning current circuit for hall measurements down to the nanotesla range," IEEE Transactions on Instrumentation and Measurement 66, 637-650 (2017).

${ }^{27} \mathrm{X}$. Hu, Y. Xu, and J. Xu, "A low-offset current-mode cmos vertical hall sensor microsystem with four-phase spinning current technique," in 2018 China Semiconductor Technology International Conference (CSTIC) (2018) pp. 1-3.

${ }^{28}$ H. Reyvandi, F. Shakibaee, and M. Saberi, "A 6-bit 100-ms/s fully-digital time-based analog-to-digital converter," (2019) pp. 412-415.

${ }^{29}$ Y. Xu, H.-B. Pan, S.-Z. He, and L. Li, "A highly sensitive cmos digital hall sensor for low magnetic field applications," Sensors (Basel, Switzerland) 12, 2162-74 (2012).

${ }^{30} \mathrm{~T}$. Watanabe and T. Terasawa, "All-digital-adc tad in sensor digitization for scaling over wide temperature ranges," (2015) pp. 1-4.

${ }^{31}$ T. Watanabe, T. Mizuno, and Y. Makino, "An all-digital analog-to-digital converter with $12-\mu \mathrm{v} / \mathrm{lsb}$ using moving-average filtering," IEEE Journal of Solid-State Circuits 38, 120-125 (2003).

${ }^{32}$ B. Kim, W. Xu, and C. Kim, "A fully-digital beat-frequency based adc achieving 39db sndr for a 1.6mvpp input signal," (2013) pp. 1-4.

${ }^{33}$ S. Huber, W. Leten, M. Ackermann, C. Schott, and O. Paul, "A fully integrated analog compensation for the piezo-hall effect in a cmos single-chip hall sensor microsystem," IEEE Sensors Journal 15, 2924-2933 (2015).

${ }^{34}$ P. Liu, K. Skucha, Y. Duan, M. Megens, J. Kim, I. Izyumin, S. Gambini, and B. Boser, "Magnetic relaxation detector for microbead labels in biomedical assays," in 2011 Symposium on VLSI Circuits - Digest of Technical Papers (2011) pp. 176-177. 